\title{
Contouring of zygomatic soft tissue using bilateral free groin flaps in a Treacher Collins syndrome patient
}

Jae-Woo Heo, Ung Sik Jin

Department of Plastic and Reconstructive Surgery, Seoul National University Hospital, Seoul National University College of Medicine, Seoul, Korea
Treacher Collins syndrome is a congenital disorder that is characterized with a wide range of cranio-facial deformities. Zygomatic hypoplasia or aplasia is one of the key features, and surgical reconstruction of the consequent depression on the zygomatic area is deemed necessary by many patients. Various surgical options are available-injectables, alloplastic materials, autologous grafting, and autogenous tissue transfer. It depends on each patient which technique to use. Here, we present a clinical case, in which bilateral free groin flaps were adopted in attempt to resolve the remnant aesthetic deformity associated with zygomatic depression, despite a series of previous surgical efforts, in a 25-year-old Treacher Collins syndrome male patient.

Keywords: Treacher Collins syndrome / Groin flaps / Free flap

\section{INTRODUCTION}

Treacher Collins syndrome is a congenital disorder with characteristic disfiguring features on craniofacial appearances. There is a gene mutation known to be responsible. However, the preliminary diagnosis is based on clinical features. Affected individuals may present with physical features such as cleft palate, micrognathia, drooping of lateral lower eyelids, microtia or anotia, zygomatic hypoplasia or aplasia and downward slanting of eyes [1]. Yet, there exists a variation from a person to person.

Breathing, feeding, seeing and hearing are the functional problems that bear a clinical significance in the Treacher Collins syndrome patients. Through staged surgical corrections, improvement of functional impairments may be achieved. However, surgeons should never forget to embrace the patient's aesthetic dissatisfaction with equivalent importance.

The methods of facial contouring can range from noninvasive

\footnotetext{
Correspondence: Ung Sik Jin

Department of Plastic and Reconstructive Surgery, Seoul National University Hospital, Seoul National University College of Medicine, 101 Daehak-ro, Jongno-gu, Seoul 03080, Korea

E-mail: usj1011@snu.ac.kr

Received January 7, 2018 / Revised March 14, 2018 / Accepted March 30, 2018
}

techniques such as filler injection to minimally invasive techniques including fat and dermofat grafting to more invasive techniques. Depending on the source of material, invasive surgical techniques can be classified into autogenous tissue or alloplastic materials. The degree and severity of volume deficit serve as the main criteria that determine which technique to use.

\section{CASE REPORT}

A 25-year-old male patient, postnatally diagnosed with Treacher Collins syndrome, presented with unsatisfactory facial appearances. The chief complaint was the depression in both zygomatic areas.

Prior to visit to our clinic, the patient underwent a series of operations at other institutions: (1) bilateral zygoma distraction osteogenesis in 2004, (2) two-jaw operation and Gore-Tex (W. L. Gore and Associates Inc., Flagstaff, AZ, USA) insertion on right zygoma in 2010, (3) bilateral coloboma correction with rotation of upper eyelid myocutaneous flap, bilateral lateral canthopexy, bilateral zygoma reconstruction with split rib bone grafting and left mandible inferior border and angle reconstruction also with rib bone grafting in 2013, and (4) dermofat and fat graft on bilateral 
zygoma areas and cheeks in 2014 (Fig. 1A). Despite previous surgeries, the patient was yet to be pleased, especially with his zygomatic depression. Significant changes in zygoma volume and contour before and after fat and dermofat grafting were not noteworthy (Fig. 1B). On July 7, 2016, another surgical correction was planned to fulfill the insufficient volume.

The patient denied of any family history. There was no significant previous medical, other operation or medication history worth of mentioning. His preoperative American Society of Anesthesiologists grading was class I. According to the preoperative physical examinations, all facial motors were normal. With exception of cutaneous sensibility decreased to 3 out of scale of 10 on both mandibular branch area, since two-jaw operation, facial sensations were normal, as well.

Bilateral free groin flaps were chosen as a mean of reconstruction. On each side of the groin, a flap with approximately $13 \times$ 6-cm-sized skin paddle was designed. Flaps were elevated with superficial circumflex iliac artery and vein. Then, bilateral preauricular incisions were made and dissection was done above Superficial Musculo-Aponeurotic System plane. Recipient vascular pedicles to be used were superficial temporal artery and vein. After de-epithelialization of both flaps, donor vascular pedicles were disconnected one flap by one. Microanastomosis was also done one flap at a time, in an end-to-end fashion, in all cases. Finally, de-epithelialized flaps were slid into each defect zone, and wound was closed by layers after insertion of a negative-pressure closedsuction drain in each side. Overall, the surgery went uneventful.

During the postoperative periods, flaps were monitored using a portal Doppler sonography to identify any abnormal blood flows. Skins above the flaps were palpated on regular basis for any increased turgor. Jackson-Pratt drains were inserted into each flap and drainages were evaluated daily. No dark sanguinous discharges were noted throughout the entire period, and both flaps remained stable without need for any surgical revisions. On the 7th day, the patient was discharged from the hospital. A 6-month follow-up showed a tolerable reconstructive result with no demonstrable signs of fat necrosis (Fig. 1C). Donor site morbidity was minimal.
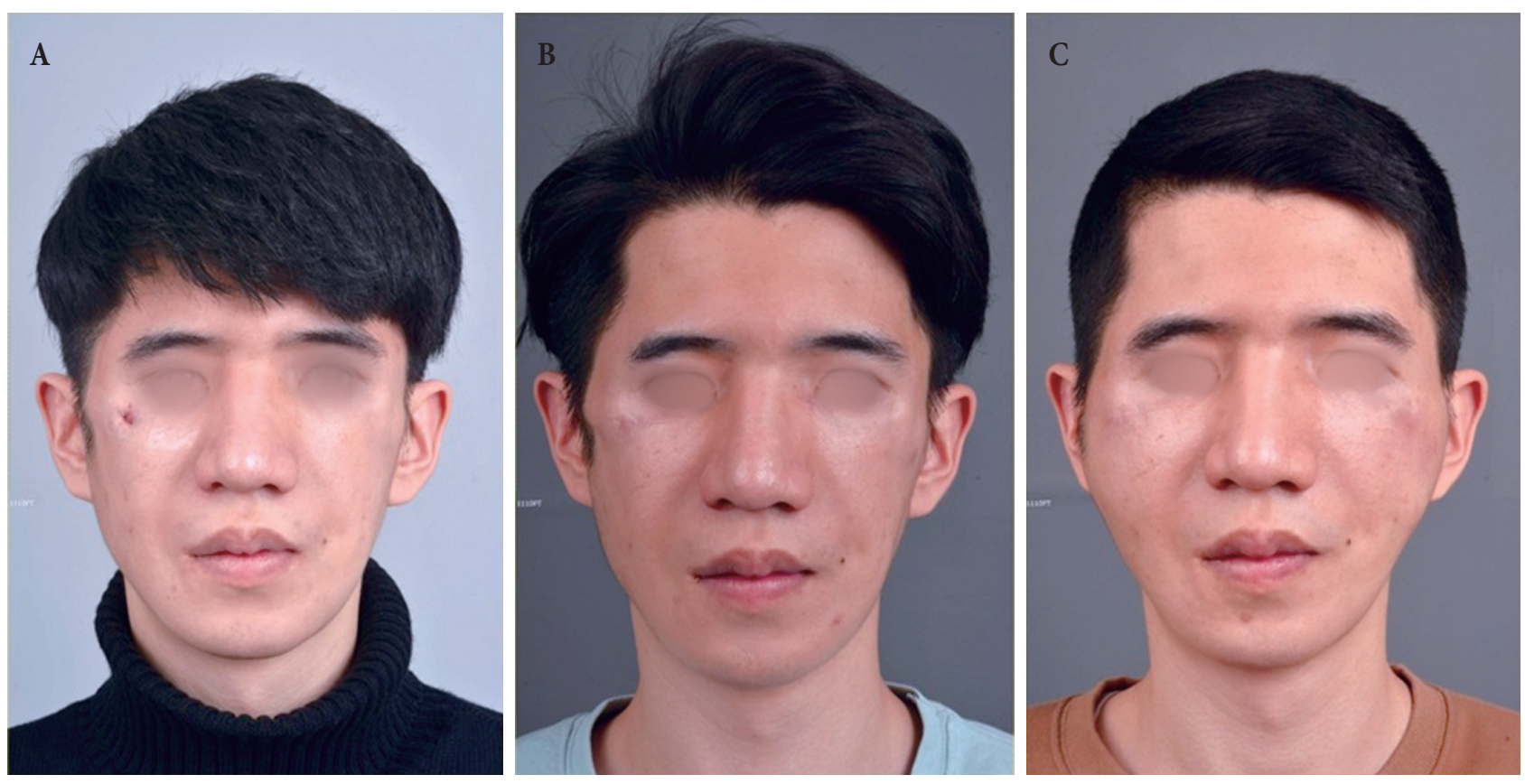

Fig. 1. (A) Photograph before fat and dermofat grafting on bilateral zygomatic areas and cheeks in July 2014. (B) Photograph before reconstruction with bilateral groin flaps on zygomatic areas and cheeks in July 2016. Note insignificant changes in zygoma volume and contour before and after fat and dermofat grafting. (C) Postoperative 6-month photograph. 


\section{DISCUSSION}

Treacher Collins syndrome is a relatively rare disorder, but once manifested, it may stigmatize affected individuals with multiple craniofacial features. Over the last few decades, many attempts have been made to come up with a both safe and efficient surgical solution. However, no golden standard technique is yet to be established, especially for volume supplementation in depressed facial areas.

In the field of plastic surgery, inadequate volume supplementation can be achieved via various methods. Filler injection and fat grafting are less invasive techniques. However, major drawback lies in a progressive resorption and a potential need for secondary touch [1]. Alloplastic materials, on the other hand, are not subject to issues regarding self-resorption; however, they are prone to inflammatory reactions or even infection, which may necessitate the undoing of the surgical procedures [2]. Reconstruction with an autogenous tissue such as dermofat is a relatively easy and costeffective surgical procedure, but, it is also not completely free from resorption [2].

This paper deals with a clinical case, in which all of the above mentioned techniques were performed for elevation of volumedeficit zygomatic area in a Treacher Collins syndrome patient. However, the main problem was a loss of bulk due to self-resorption after autologous grafting. Off note, there also were problems concerning inflammation and infection after the use of alloplastic materials. As a result, the use of tissue transfer was taken into a consideration.

Flaps can provide a long-lasting or even permanent outcome. Pedicled flaps may be of a little use in facial contouring due to pedicle length and restriction in flap size. Vascularized free flaps, depending on their donor sites, can provide an appropriate bulk that the patient desires.

Various free flaps have been adopted in attempt for facial contouring. In comparison to other flaps, groin flaps require a less elaborate preoperative planning, and the operation time is relatively short [3]. The greatest virtue lies in the acceptable donor site morbidity; scar itself can be concealed, primary donor site closure is almost always possible thanks to abundant groin skin, and functional impairment is less likely except for limitation of ambulation during immediate postoperative periods $[4,5]$. Furthermore, especially if the volume deficit is bilateral as in this case, there are always two symmetric donor sites to use.

Nevertheless, selecting vascularized flaps primarily above other methods may not be a wise solution, all the time-long operation time, donor site morbidity, risk of threatened flap survival, etc. Yet, with all the less invasive technique having failed to meet the patient's expectations, as in this case, free flap reconstruction should be considered the next option.

Reconstruction using a free flap may be the final salvage after all other methods having failed to meet the patient's satisfaction. And, in circumstances where the facial volume deficit is mild to moderate in degrees, as in this case, groin flaps have proven to be an adequate method. However, whether to use free flaps from the start is a question yet to be fully answered. Operators' surgical skills and patient's personal wants should be weighed and balanced to choose appropriate reconstruction method. However, with the unprecedented development of microsurgical tools and skills, and minimal concerns for self-resorption and infection, free flaps may be considered as a primary solution to facial volume deficit.

\section{CONFLICT OF INTEREST}

No potential conflict of interest relevant to this article was reported.

\section{PATIENT CONSENT}

The patients provided written informed consent for the publication and the use of their images.

\section{REFERENCES}

1. Rodriguez ED, Losee JE, Nelligan PC. Plastic surgery. Vol. 3: craniofacial, head and neck surgery pediatric plastic surgery. New York: Elsevier Health Sciences; 2012.

2. Mordick TG 2nd, Larossa D, Whitaker L. Soft-tissue reconstruction of the face: a comparison of dermal-fat grafting and vascularized tissue transfer. Ann Plast Surg 1992;29:390-6. 
3. Dunkley MP, Stevenson JH. Experience with the free "inverted" groin flap in facial soft tissue contouring: a report on 6 flaps. Br J Plast Surg 1990;43:154-8.
4. McGregor IA, Jackson IT. The groin flap. Br J Plast Surg 1972;25:3-16.

5. Harashina T, Fujino T. Reconstruction in Romberg's disease with free groin flap. Ann Plast Surg 1981;7:289-94. 